\title{
Development of Polysulfone/Activated Carbon Nanofibers Mixed Matrix Membrane for $\mathrm{CO}_{2} / \mathrm{CH}_{4}$ Separation
}

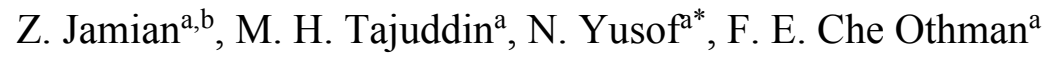 \\ aAdvanced Membrane Technology Research Centre (AMTEC), Universiti Teknologi \\ Malaysia, 81310 UTM Johor Bahru, Johor, Malaysia \\ ${ }^{\mathrm{b}}$ Gas Malaysia Berhad (Pasir Gudang) 81700 Pasir Gudang, Johor, Malaysia
}

Submitted: 26/4/2018. Revised edition: 15/5/2018. Accepted: 24/5/2018. Published online: 4/6/2018

\begin{abstract}
This study was performed primarily to investigate the effect of activated carbon nanofiber (ACNF) on carbon dioxide and methane separation performance of mixed matrix membrane (MMM). In this study, polysulfone (PSf)/ACNF mixed matrix membranes was fabricated using dry/wet inversion technique. The effect of PSf concentration and ACNF loading on the performance of mixed matrix membrane in terms of permeability and selectivity of $\mathrm{CO}_{2} / \mathrm{CH}_{4}$ gas separation was observed. The fabricated flat sheet mixed matrix membranes were characterized using Fourier Transform Infrared Spectroscopy (FT-IR) and Scanning Electron Microscopy (SEM) analysis. From the SEM observations, it shows that sponge like structures images were observed upon the addition of ACNFs in the PSf/ACNF membranes was slowly decreased due to increasing weight percentage of ACNF. FT-IR result indicating the presence of carboxyl group in MMM at wavelength $1750 \mathrm{~cm}^{-1}$. Meanwhile, the MMMs were further tested to pure permeation test using pure $\mathrm{CO}_{2}$ and $\mathrm{CH}_{4}$ gas, the $\mathrm{CO}_{2}$ permeance improved and the selectivity of $\mathrm{CO}_{2} / \mathrm{CH}_{4}$ increased after the addition of ACNFs.
\end{abstract}

Keywords: Activated carbon nanofiber, mixed matrix membrane, gas separation

\subsection{INTRODUCTION}

It is attractive to evacuate non-fuel gasses with a specific end goal to minimize operation costs and expand the heating value of the gas. Acid gasses, for example, carbon dioxide and hydrogen sulfide, must be expelled to avert consumption in pipelines and handling equipment. Carbon dioxide is the most bottomless contaminant in numerous gas stores, and by far most of regular gas separation look into has been committed to its evacuation [1]. As characteristic of natural gas request increments over the coming years, more effective separation innovations that permit expanded throughput with negligible handling expense was important to keep normal gas costs manageable.

These days, there are a few set up procedures that have been presently connected to address this issue, for example, amine based absorption, potassium carbonate based absorption, cryogenic separation process, and membranes separation technology. [2] Each of the procedures offers diverse division components with specific favorable circumstances and limitations. The most broadly utilized procedure to sanitize natural gas uses alkanolamine aqueous solution to absorb specifically carbon dioxide and hydrogen sulphite from natural gas. This procedure is to great degree energy intensive and requires different steps arrangement for the separation and solution recovery including 
heating of the recovery acid gas [3]

Polymeric membranes are the most widely recognized membranes for gas separation process. There are already membrane systems installed for natural gas sweetening many places in the world, including offshore Malaysia [4] However, the performances of polymeric membrane are limited to low productivity and trade off effects between selectivity/permeability. To address this issue, the development of mixed matrix membrane (MMM) membranes is proposed to overcome the limitation of polymeric membranes [5]. Combination of the organic (polymer) with inorganic materials give birth to a nanocomposite membranes which will possess the synergistic advantages from each phase: high selectivity and permeability of the dispersed fillers and easy processability of the polymers [6].

Herein, in this study we proposed $\mathrm{ACNF}$ to be incorporated in PSf membrane to study the effect of its physicochemical properties and their gas separation performances.

\subsection{METHODS}

\subsection{Materials}

Commercially available Polysulfone pellets (PSF;Udel P3500) were purchased from Solvay polymers as the membrane substrates polymer and 1,2methyl pyrolidonne (NMP) and tetrahydrofuran (THF) were purchased from Merck as the solvent to dissolve the polymers. ACNF were produced by pyrolysis of NFs from polyacrylonitrile. The NFs were later carbonized at $600^{\circ} \mathrm{C}$ under nitrogen flow at heating rate of $5^{\circ} \mathrm{Cmin}^{-1}$ to produce carbon nanofibers (CNFs). The CNFs were physically activated in horizontal tube furnace (Carbolite
CTF12/65/550) at temperature of $800^{\circ} \mathrm{C}$ under carbon dioxide flow at heating rate of $5^{\circ} \mathrm{C} \mathrm{min}^{-1}$ to yield activated carbon nanofibers (ACNFs) [6].

\subsection{Preparation of Mixed Matrix Membrane}

The fabrication of neat PSf and MMMs were adapted from Nordin et al. [7]. PSf pellets $(25 \mathrm{wt} \%)$ was dissolved in NMP (37.5 wt\%) and THF (37.5 wt $\%)$ solution at the room temperature for at least $12 \mathrm{~h}$ until the solution become homogeneous. For preparation of PSf/ACNFs MMMs, the loading of ACNFs was varied in a range from $0.0 \mathrm{wt} \%$ to $1.5 \mathrm{wt} \%$ per total weight of polymer. Table 1 referred to the summarized concentration used in this study. The ACNF powder were added in NMP $(37.5 \mathrm{wt} \%)$ and THF $(37.5 \mathrm{wt} \%)$ solution and stirred for 5 hours until the solution become homogenous. The PSf pellets were subsequently added in the mixture solution and stirred on the hot plate for at least 12 hours until the dope solution became homogenous. The dope solution was degassed at the room temperature to remove all air bubbles.

Table 1 Summarized concentrations for dope solution

\begin{tabular}{cllll}
\hline Sample & $\begin{array}{c}\text { PSf } \\
(\mathbf{w t} \%)\end{array}$ & $\begin{array}{c}\text { NMP } \\
(\mathbf{w t} \%)\end{array}$ & $\begin{array}{c}\text { THF } \\
(\mathbf{w t} \%)\end{array}$ & $\begin{array}{c}\text { ACNF } \\
(\mathbf{w t} \%)\end{array}$ \\
\hline 1 & 25 & 37.5 & 37.5 & 0.0 \\
2 & 25 & 37.5 & 37.5 & 0.1 \\
3 & 25 & 37.5 & 37.5 & 0.5 \\
4 & 25 & 37.5 & 37.5 & 1.0 \\
5 & 25 & 37.5 & 37.5 & 1.5 \\
\hline
\end{tabular}

A flat sheet membrane was prepared via dry/wet inversion technique using manual casting method. The dope solution was casted 
on clean glass plate to form thin film $0.1 \mathrm{~cm}$. After standing on the glass plate for approximately $3 \mathrm{~min}$, the casted layer and the glass plate was immersed in a coagulation bath (deionized water) at room temperature where the membrane was solidified. After that the membranes was stored in fresh water bath for at least 1 day to leach out the residual solvents completely. Last step, the membrane was dried for 24 hours in the ambient atmosphere. The other membrane was prepared by the same process for sample 2, 3, 4 and 5 respectively as shown in the Figure 1.
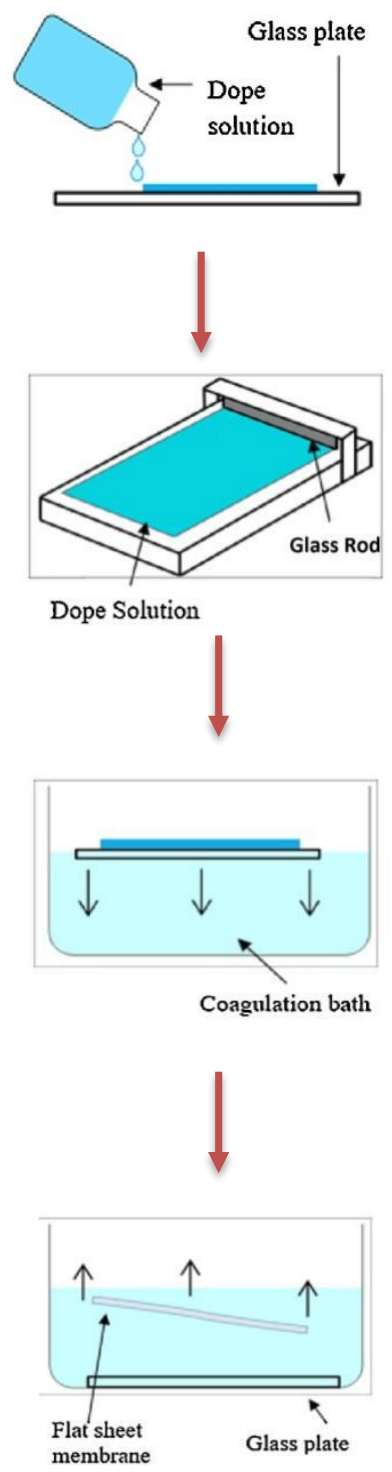

Figure 1 Steps in fabrication of flat sheet membrane

\subsection{Characterization of $M M M s$}

The neat membrane and MMMs was further characterized to understand their physicochemical properties. The membranes morphological structures were characterized by Scanning Electron Microscopy (SEM) with magnification $3.0 \mathrm{k}$ and $5.0 \mathrm{k}$ analysis with an accelerating voltage of 20.0 $\mathrm{kV}$. Chemical functional group of neat and MMMs were analysed by using FTIR (Fourier Transform Infrared Spectroscopy) at wavelength about 400 $\mathrm{cm}^{-1}$ to $4000 \mathrm{~cm}^{-1}$ and $0.25 \mathrm{~cm}^{-1}$ scanning resolution has been set.

\subsection{Gas Permeation Test}

Gas permeation system was made up to measure pure gas permeation rate for asymmetric flat sheet membrane and approximately their selectivity. Figure 2 illustrated the overall gas permeation system used in this study. Gas permeation test was prepared using two high purity (99.99\%) of gas which is $\mathrm{CO}_{2}$ and $\mathrm{CH}_{4}$. The main objective is to determine the level of permeability of membrane based on volume displacement method at room temperature and low operating pressure. The permeation coefficient was determined by apply the following equation:

$\mathrm{P}_{\mathrm{i}}=\mathrm{Q}_{\mathrm{i}} \times \mathrm{L} / \mathrm{A} \times \Delta \mathrm{P}$

where $P_{i}$ is the permeability of gas permeation through the membrane which is expressed in GPU (1 GPU = $\left.10^{-6} \mathrm{~cm}^{3} \mathrm{STP} / \mathrm{cm}^{2} \mathrm{~s} \mathrm{~cm} \mathrm{Hg}\right) ; \mathrm{Q}_{i}$ is volumetric gas flowrate of gas $\mathrm{CO}_{2}$ and $\mathrm{CH}_{4}\left(\mathrm{~cm}^{3} / \mathrm{s}\right) ; \quad \backslash \mathrm{P}$ is pressure difference across membrane $(\mathrm{cm} \mathrm{Hg})$; $\mathrm{L}$ is thickness of membrane $(\mathrm{cm})$ and $A$ is effective membrane for permeation test $\left(\mathrm{cm}^{2}\right)$. 
Selectivity of prepared membrane was determined using following equation.

$\alpha=\mathrm{P}_{\mathrm{i}} / \mathrm{P}_{\mathrm{j}}$

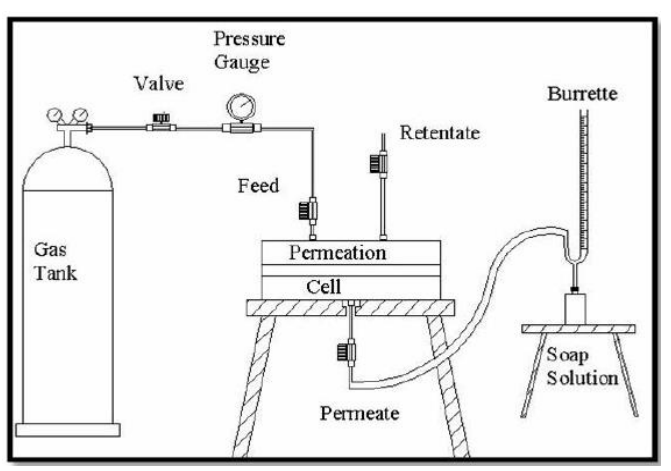

Figure 2 Gas permeation system

\subsection{RESULTS AND DISCUSSION}

\subsection{Functional Group Analysis}

The prepared neat PSF and ACNF MMMs were characterized by Fourier Transform Infrared Spectroscopy (FTIR) to identify chemical functional groups of fabricated membranes. Figure 3 illustrates the stretching band of MMMs which made by using various $0.0 \mathrm{wt} \%, \quad 0.1 \mathrm{wt} \%, \quad 0.5 \mathrm{wt} \%$, $1.0 \mathrm{wt} \%$ and $1.5 \mathrm{wt} \%$ of ACNF respectively. There are bands at around $1107-1092 \mathrm{~cm}^{-1}$ that indicated for the aromatic ring vibrations. The strong absorptions due to the asymmetric stretches of sulfone group in PSf molecule were found at round that range belong to the aromatic $\mathrm{O}=\mathrm{S}=\mathrm{O}$ in polysulfone molecules. $\mathrm{C}-\mathrm{H}$ stretching band sample that were detected at frequency around 3200$2800 \mathrm{~cm}^{-1}$ [8-9]. Membrane fabricated from mixture of $\mathrm{PSf} / \mathrm{NMP} / \mathrm{ACNF}$ showed positive attending of carboxylic group asymmetric $\mathrm{C}=\mathrm{O}$ that stretching of carboxylic acid group at frequency around $1780-1710 \mathrm{~cm}^{-1}$. From the findings, it shows that the chemical functional that appears probably contributed to excellent permeability and selectivity.

\subsection{Morphology and Structure of PSf/ACNF MMMs}

The morphological structures of neat PSf and MMMs were analysed by using SEM analysis. As can be seen cross section images from Figure 4 (ae), From the SEM images, all membranes exhibited partially sponge type porous structures. The porous structures of MMMs were slowly decreasing upon the addition of ACNFs as presented in Figure $4(\mathrm{~g}-\mathrm{j})$. However, the presence of ACNFs are not detected by SEM images probably attributed to smaller size of ACNFs and well-distrbution of this fillers in membrane matrix [10]. Meanwhile, Figure $4(f-j)$ displayed the surface micrographs of neat membrane and ACNFs composite membranes. From the SEM images, all membranes show that pinhole appeared on the surface and decreasing as the loading of ACNFs is increasing. The appearances of pinhole on these surfaces probably contributed by non-uniform distribution of ACNFs in the membranes structures. However, as the loading of ACNFs is increasing the pinhole on the surface is slowly diminished.

\subsection{Gas Separation Performance}

To observe the effect of ACNFsMMMs were further investigated by the gas separation performances methane and carbon dioxide. Based on the Table 2 presented below, shows the selectivity and permeability of membrane has improved as the loading of ACNFs is increased. The $\mathrm{CO}_{2}$ permeability and $\mathrm{CO}_{2} / \mathrm{CH}_{4}$ selectivity of neat PSf are 20.51 GPU and 1.32 respectively. The lower permeability value and selectivity probably attributed to the different materials and fabrication methods

[7]. 


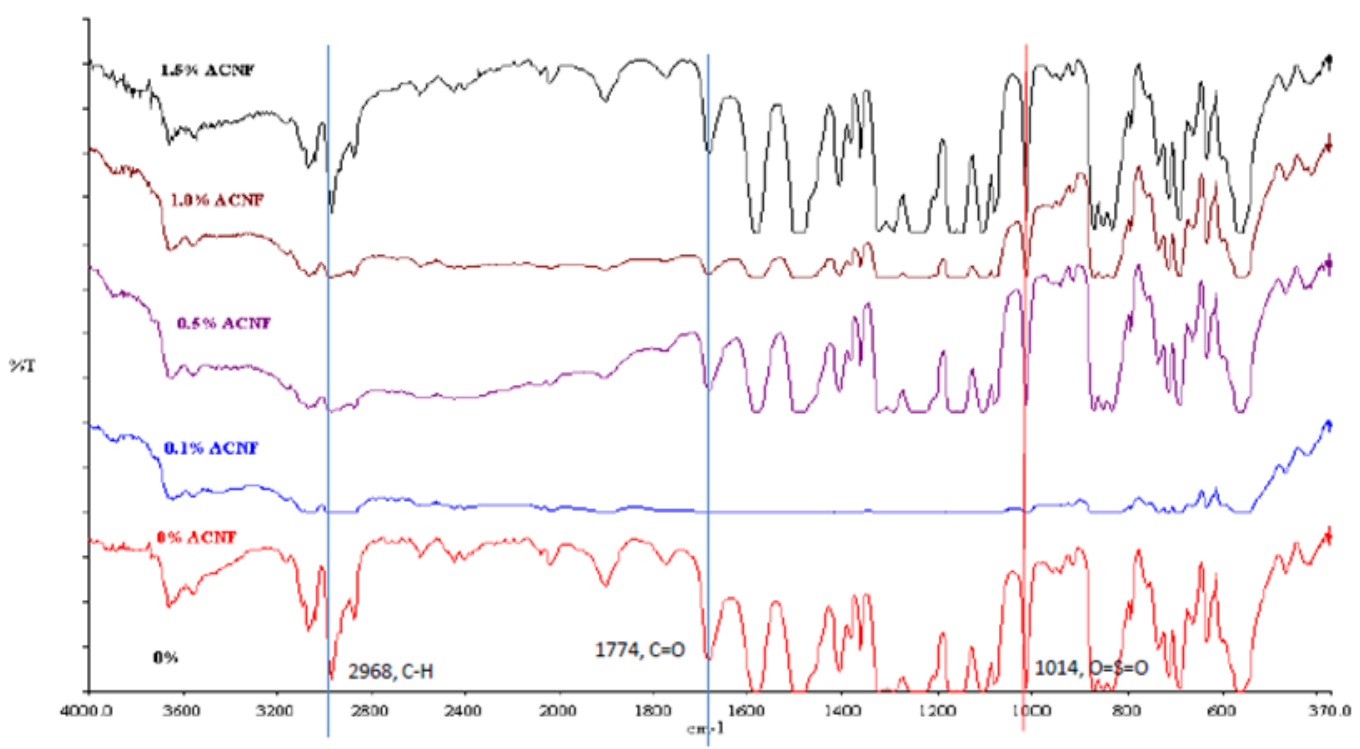

Figure 3 FTIR spectrums of neat membrane and MMMs membranes
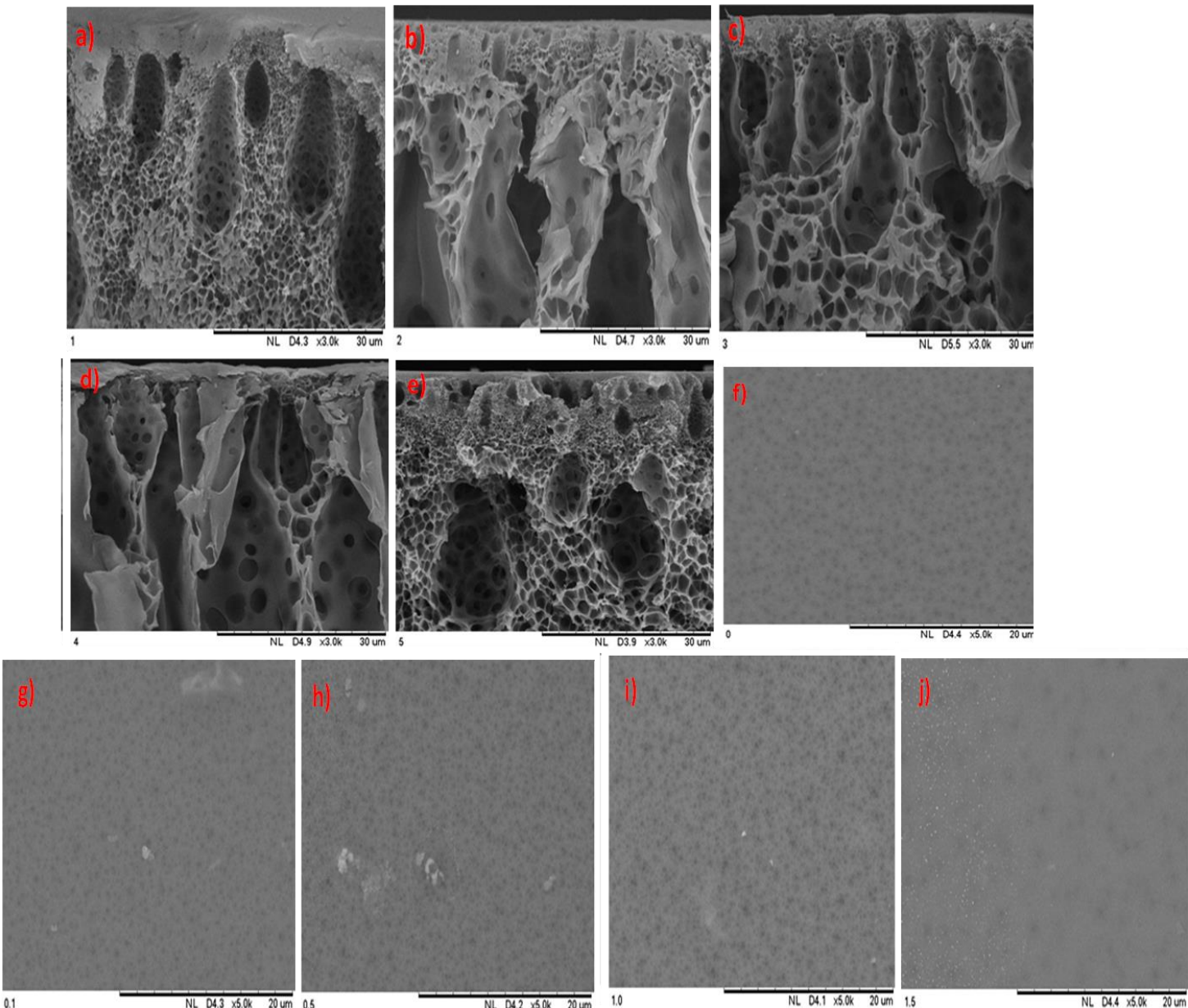

Figure 4 SEM images of cross-sections of polysulfone containing different concentration of ACNF (a) $0.0 \mathrm{wt} \%$, (b) $0.1 \mathrm{wt} \%$, (c) $0.5 \mathrm{wt} \%$, (d) $1.0 \mathrm{wt} \%$, (e) $1.5 \mathrm{wt} \%$ and surface images of polysulfone containing different concentration of ACNF (f) $0.0 \mathrm{wt} \%$, (g) $0.1 \mathrm{wt} \%$, (h) $0.5 \mathrm{wt} \%$, (i) $1.0 \mathrm{wt} \%$, (j) $1.5 \mathrm{wt} \%$ 
Table 2 Permeability and selectivity of PSf and MMMs

\begin{tabular}{|c|c|c|c|}
\hline \multirow[t]{2}{*}{ Sample } & \multicolumn{2}{|c|}{ Permeability (GPU) } & \multirow{2}{*}{$\begin{array}{c}\text { Selectivity } \\
\mathrm{CO}_{2} / \mathrm{CH}_{4}\end{array}$} \\
\hline & $\mathrm{CO}_{2}$ & $\mathrm{CH}_{4}$ & \\
\hline Neat PSf & 20.51 & 15.55 & 1.32 \\
\hline $0.1 \mathrm{wt} \%$ ACNFs -MMMs & 22.45 & 16.39 & 1.37 \\
\hline $0.5 \mathrm{wt} \%$ ACNFs -MMMs & 26.35 & 18.82 & 1.40 \\
\hline $1.0 \mathrm{wt} \%$ ACNFs -MMMs & 30.81 & 21.00 & 1.41 \\
\hline $1.5 \mathrm{wt} \%$ ACNFs -MMMs & 35.26 & 24.67 & 1.43 \\
\hline
\end{tabular}

Upon the addition of ACNFs in polymer matrix, the $\mathrm{CO}_{2}$ permeability and $\mathrm{CO}_{2} / \mathrm{CH}_{4}$ selectivity are slowly increased compared with neat PSf. [7]. The enhancement in permeability were assumed caused by the interaction between polymer and ACNFs that could distort the polymer chain packing thus resulting in increased of gas diffusion due to more free volumes introduced [10]. Furthermore, the $\mathrm{CO}_{2} / \mathrm{CH}_{4}$ selectivity increased after the addition of ACNFs. The increased in selectivity might be related with the functional group such $\mathrm{C}=\mathrm{O}$ in ACNFs as presented in FTIR results. The carboxyls group has stronger attraction with polar gas such as carbon dioxide that caused the solubity and gas permeability of $\mathrm{CO}_{2}$ increased [11-12]

\subsection{CONCLUSION}

MMMs deriving from ACNF with different composition fabricated via dry/wet inversion technique. The SEM images obtained the cross-sectional area of the PSf/ACNF membranes indicated that ACNFs well dispersed in the PSF matrix at 0.1 , and $1.5 \mathrm{wt} \%$ ACNF loading. Based on the results, shown that the performance of the MMMs have relied on the precursor polymer composition. Furthermore, the PSf/ACNF MMMs selectivity and permeability increased slightly to create best composite membrane.

\section{ACKNOWLEDGEMENT}

The author would like to acknowledge the financial support from the Ministry of Higher Education Malaysia and Universiti Teknologi Malaysia under Prototype Development Research Grant Scheme - ICC (R.J130000.7746.4F929) and Research University (Q.J130000.2546.12H54) and Gas Malaysia Berhad.

\section{REFERENCES}

[1] S. Anastasiou et al. 2018. Metalorganic Framework/graphene Oxide Composite Fillers in Mixed-matrix Membranes for $\mathrm{CO}_{2}$ Separation. Mater. Chem. Phys. 212: 513-522.

[2] O. Bakhtiari and N. Sadeghi. 2014. Mixed Matrix Membranes Gas Separation Performance Prediction Using an Analytical Model. Chem. Eng. Res. Des. 3: 710-719.

[3] M. Rezakazemi, M. Sadrzadeh, and T. Matsuura. 2018. Thermally Stable Polymers for Advanced High-performance Gas Separation Membranes. Prog. Energy Combust. Sci. 66. $1-41$.

[4] M. Vinoba, M. Bhagiyalakshmi, Y. Alqaheem, A. A. Alomair, A. Pérez, and M. S. Rana. 2017. Recent Progress of Fillers in Mixed Matrix Membranes for 
CO2 Separation: A Review. Sep. Purif. Technol. 188: 431-450.

[5] D. Tutuk, F. Ahmad, and M. Ahmad. 2010. Application of Activated Carbon Mixed Matrix Membrane for Oxygen Purification. Int. J. 1: 21-24.

[6] F. E. Che Othman et al. 2017. Polyacrylonitrile/magnesium Oxide-based Activated Carbon Nanofibers with Well-developed Microporous Structure and Their Adsorption Performance for Methane. J. Ind. Eng. Chem. 51: 281-287.

[7] N. A. H. Md Nordin et al. 2015. Facile Modification of ZIF-8 Mixed Matrix Membrane for $\mathrm{CO}_{2} / \mathrm{CH}{ }_{4}$ Separation: Synthesis and Preparation. RSC Adv. 5: 43110-43120.

[8] A. D. Kiadehi, A. Rahimpour, M. Jahanshahi, and A. A. Ghoreyshi. 2015. Chemistry Novel Carbon Nano-fibers (CNF)/polysulfone (PSf) Mixed Matrix Membranes For Gas Separation. J. Ind. Eng. Chem. 22: 199-207.
[9] M. S. Boroglu and A. B. Yumru. 2017. Gas Separation Performance of 6FDA-DAMZIF-11 Mixed-matrix Membranes for $\mathrm{H}_{2} / \mathrm{CH}_{4}$ and $\mathrm{CO}_{2} / \mathrm{CH}_{4}$ Separation. Sep. Purif. Technol. 173 . 5130-5132.

[10] A. D. Kiadehi, M. Jahanshahi, A. Rahimpour, and S. A. A. Ghoreyshi. 2015. Process Intensi fication the Effect of Functionalized Carbon Nano-fi ber (CNF) on Gas Separation Performance of Polysulfone (PSf) Membranes. Chem. Eng. Process. Process Intensif. 90: 41-48.

[11] A. Torkkeli. 2003. Droplet Microfluidics on a Planar Surface. VTT Publ. 53: 3-194.

[12] P. Hrabanek, R. Mallada, and V. Fila. 2017. Study of Different Titanosilicate (TS-1 and ETS-10) as Fillers for Mixed Matrix Membranes for $\mathrm{CO}_{2} / \mathrm{CH}_{4}$ Gas Separation Applications. $J$. Memb. Sci. 523: 24-35. 